\title{
TANGENTIAL BEHAVIOR OF FUNCTIONS AND CONICAL DENSITIES OF HAUSDORFF MEASURES
}

\author{
VILLE SUOMALA
}

\begin{abstract}
We construct a $C^{1}$-function $f:[0,1] \rightarrow \mathbb{R}$ such that for almost all $x \in(0,1)$, there is $r>0$ for which $f(y)>f(x)+f^{\prime}(x)(y-x)$ when $y \in(x, x+r)$ and $f(y)<f(x)+f^{\prime}(x)(y-x)$ when $y \in(x-r, x)$. The existence of such functions is related to a problem concerning conical density properties of Hausdorff measures on $\mathbb{R}^{n}$. We also discuss the tangential behavior of typical $C^{1}$-functions, using an improvement of Jarnik's theorem on essential derived numbers.
\end{abstract}

\section{INTRODUCTION AND NOTATION}

Let us begin by introducing some notation. For $0 \leq s \leq n$, let $\mathcal{H}^{s}$ denote the $s$-dimensional Hausdorff measure on $\mathbb{R}^{n}$, and on the real line, let $\mathcal{L}$ stand for the Lebesgue measure. We use the common notation $B(x, r)$ for open balls on Euclidean and metric spaces, and for the unit sphere on $\mathbb{R}^{n}$ the notation $S^{n-1}=\left\{x \in \mathbb{R}^{n}:|x|=1\right\}$ is used. If $x \in \mathbb{R}^{n}$, and $A \subset \mathbb{R}^{n}$, then $d(x, A)$ stands for the Euclidean distance between $x$ and $A$. The length of an interval $I \subset \mathbb{R}$ is denoted by $\ell(I)$ and the notion $\partial A$ is used for the boundary of a given set $A \subset \mathbb{R}$. The (symmetrical) upper and lower densities of $A \subset \mathbb{R}$ at $x \in \mathbb{R}$ are defined as the upper and lower limits, respectively, of the ratio $\mathcal{L}((x-r, x+r) \cap A) /(2 r)$ when $r \downarrow 0$. If $f:[0,1] \rightarrow \mathbb{R}$ is differentiable at $x \in(0,1)$, the sets $A^{+}(f, x)$ and $A^{-}(f, x)$ are given by

$$
\begin{aligned}
& A^{+}(f, x)=\left\{y \in(0,1): f(y)>f(x)+f^{\prime}(x)(y-x)\right\}, \\
& A^{-}(f, x)=\left\{y \in(0,1): f(y)<f(x)+f^{\prime}(x)(y-x)\right\} .
\end{aligned}
$$

For $r>0$, we put $A^{+}(f, x, r)=A^{+}(f, x) \cap(x-r, x+r)$ and $A^{-}(f, x, r)=$ $A^{-}(f, x) \cap(x-r, x+r)$. If $x \in \mathbb{R}^{n}, \theta \in S^{n-1}$, and $0 \leq \eta<1$, we define the cone $H(x, \theta, \eta)$ by setting $H(x, \theta, \eta)=\left\{y \in \mathbb{R}^{n}:(y-x) \cdot \theta>\eta|y-x|\right\}$. For half-spaces we use shorter notation, $H(x, \theta)=H(x, \theta, 0)$.

Date: 13 th August 2004.

2000 Mathematics Subject Classification. 26A24, 28A78.

Key words and phrases. Conical density, essential derived number.

The author was supported by the foundation Vilho, Yrjö and Kalle Väisälä Fund, and by the Finnish graduate school in mathematical analysis. 
Given a set $A \subset \mathbb{R}^{n}$, it is often of interest to know how it is distributed near a "generic" point. This paper was inspired by the following conical density theorem of Marstrand [4, pp. 293-297].

Theorem 1.1. Let $0 \leq s<2$ and $A \subset \mathbb{R}^{2}$ with $\mathcal{H}^{s}(A)<\infty$.

(1) If $0 \leq s<1$ and $\theta \in S^{1}$, then $\liminf _{r \downarrow 0} r^{-s} \mathcal{H}^{s}(B(x, r) \cap H(x, \theta) \cap A)=0$ for $\mathcal{H}^{s}$-almost all $x \in A$.

(2) If $1<s<2$, then for $\mathcal{H}^{s}$-almost all $x \in A$, there is $\theta \in S^{1}$ such that $\liminf _{r \downarrow 0} r^{-s} \mathcal{H}^{s}(B(x, r) \cap H(x, \theta) \cap A)=0$.

It seems that 1 -sets ( $\operatorname{sets} A$ with $0<\mathcal{H}^{1}(A)<\infty$ ) play a special role in connection with the above theorem. Marstrand's proof yields that claim (2) is valid also for 1 -sets if half-spaces $H(x, \theta)$ are replaced by cones $H(x, \theta, \eta)$ for any $\eta>0$. On the other hand, Besicovitch [1, Theorem 13] had shown before that even (1) holds for purely unrectifiable 1-sets, that is, for 1-sets which intersect every rectifiable curve only in a set of zero $\mathcal{H}^{1}$-measure. A question arises whether (2) actually holds for all 1-sets.

The answer to the above question is negative. In Section 2.1 we construct a $C^{1}$-function $f:[0,1] \rightarrow \mathbb{R}$ whose graph does not satisfy claim (2) of Theorem 1.1. In Section 2.2, inspired by our example, we study the distribution of the sets $A^{+}(f, x)$ and $A^{-}(f, x)$ for functions $f \in C^{k}[0,1], k \in \mathbb{N}$. We show that for a typical $f \in C^{1}[0,1]$, both of the sets $A^{+}(f, x)$ and $A^{-}(f, x)$ have zero lower density and unit upper density at $x$ for almost every $x \in(0,1)$. This is a corollary to an extension of Jarnik's theorem on essential derived numbers (Theorem 2.3).

Finally, in Section 3, we discuss the above conical density problem in higher dimensions.

Acknowledgements. I am grateful to Professors Pertti Mattila, Luděk Zajíček, and Clifford Weil for useful discussions, comments, and suggestions. I also thank Mika Leikas, Jouni Parkkonen, and Juhani Takkinen.

\section{TANGential Behavior OF FUnCtions}

2.1. An example. Our goal in this section is to prove the following.

Theorem 2.1. There is a continuously differentiable function $f:[0,1] \rightarrow \mathbb{R}$ such that for almost all $x \in(0,1)$, there is $r>0$ for which

$$
\begin{aligned}
& (x, x+r) \subset A^{+}(f, x), \\
& (x-r, x) \subset A^{-}(f, x) .
\end{aligned}
$$

Remarks. It is quite easy to construct functions $f \in C^{\infty}[0,1]$ so that $(2.1)$ and (2.2) are valid in a set of positive measure: Define $g \in C^{\infty}[0,1]$ so that $g=0$ on some Cantor set $C$ with $\mathcal{L}(C)>0$, and $g>0$ outside this set. If $f$ is given by $f(x)=\int_{t=0}^{x} g(t) d t$, then (2.1) and (2.2) hold for $f$ on the set $C$. However, for any 
$C^{2}$-function, these conditions can not hold almost everywhere, see Proposition $2.2(1)$.

Proof of Theorem 2.1. The idea is to iterate the construction suggested by the above remark. Choose a sequence of numbers $0<\lambda_{i}<1$ for $i \in \mathbb{N}$ such that

$$
\prod_{i=1}^{\infty}\left(1-\lambda_{i}\right)>0 .
$$

Let $R_{1}=1$ and define numbers $R_{i}$ for every $i \in \mathbb{N}$ by letting $R_{i+1}=\left(1-\lambda_{i}\right) R_{i} / 2$. We define a Cantor set $C_{1} \subset(0,1)$ in a usual manner. Start by setting $Q_{1,1}=$ $\left(0,1 / 2-\lambda_{1} / 2\right), I_{1,1}=\left[1 / 2-\lambda_{1} / 2,1 / 2+\lambda_{1} / 2\right]$, and $Q_{1,2}=\left(1 / 2+\lambda_{1} / 2,1\right)$. If open intervals $\left\{Q_{i, j}\right\}_{j=1}^{2^{i}}$ and closed intervals $\left\{I_{i, j}\right\}_{j=1}^{2^{i-1}}$ have been defined, remove from the middle of every $Q_{i, j}$ a closed interval of length $\lambda_{i+1} R_{i+1}$. Denote the deleted intervals by $\left\{I_{i+1, j}\right\}_{j=1}^{2^{i}}$ and the remaining open intervals by $\left\{Q_{i+1, j}\right\}_{j=1}^{2^{i+1}}$. For every $i \in \mathbb{N}$, the length of the intervals $Q_{i, j}$ is $R_{i+1}$ and the length of the intervals $I_{i, j}$ is $\lambda_{i} R_{i}$. We set $C_{1}=(0,1) \backslash \bigcup_{i, j} I_{i, j}=\bigcap_{i} \bigcup_{j} Q_{i, j}$. It follows from (2.3) that $\mathcal{L}\left(C_{1}\right)>0$. Then we define $g_{1}(x)=d\left(x, C_{1}\right)$ for $0<x<1$ and further $f_{1}(x)=\int_{0}^{x} g_{1}(t) d t$ for any $0 \leq x \leq 1$. It is clear that $f_{1}$ is strictly monotone on $[0,1]$ and because $f_{1}^{\prime}(x)=0$ for every $x \in C_{1}$, it follows that (2.1) and (2.2) are valid for $f_{1}$ if $x \in C_{1}$. As seen above, the set $(0,1) \backslash C_{1}$ can be written as $(0,1) \backslash C_{1}=\bigcup_{i, j} I_{1, i, j}$, where intervals $I_{1, i, j}$ are closed and disjoint.

Let us continue by induction. Suppose that a function $g_{k}$ and a set $C_{k}=$ $(0,1) \backslash \bigcup_{i, j} I_{k, i, j}$ have been defined. Denote by $I_{k, i, j}^{\prime}$ the open interval with same center as $I_{k, i, j}$ and with length $\ell\left(I_{k, i, j}\right) / 2$. First we modify $g_{k}$ on each $I_{k, i, j}$ so that it remains continuous, becomes constant $\left(=b_{k, i, j}\right)$ on $I_{k, i, j}^{\prime}$, and affine on both components of $I_{k, i, j} \backslash I_{k, i, j}^{\prime}$. Denote this new function by $\widetilde{g_{k}}$ (see Figure 1). The above constant, $b_{k, i, j}$, is defined so that $\int_{I_{k, i, j}}\left(g_{k}-\widetilde{g_{k}}\right)=a_{k, i, j}$, where the constants $a_{k, i, j}>0$ are chosen sufficiently small (see the discussion in the next paragraph). Now we define $g_{k+1}$ on $I_{k, i, j}^{\prime}$ in a corresponding manner as $g_{1}$ was defined on $(0,1)$ (see Figure 1): Let $I_{k, i, j}^{\prime}=(\alpha, \beta)$. For each $x \in I_{k, i, j}^{\prime}$, we set

$$
g_{k+1}(x)=b_{k, i, j}+c_{k, i, j} g_{1}\left(\frac{x-\alpha}{\beta-\alpha}\right)
$$

where constants $c_{k, i, j}$ are chosen so that $\int_{I_{k, i, j}} g_{k+1}=\int_{I_{k, i, j}} g_{k}$. On $(0,1) \backslash \bigcup_{i, j} I_{k, i, j}^{\prime}$ we set $g_{k+1}=\widetilde{g}_{k}$. Let $C_{k+1}=\left(\bigcup_{i, j}\left(\left\{\alpha_{k, i, j}\right\}+\ell\left(I_{k, i, j}^{\prime}\right) C_{1}\right)\right) \bigcup C_{k}$, where $\alpha_{k, i, j}$ is the left endpoint of $I_{k, i, j}^{\prime}$, and write $(0,1) \backslash C_{k+1}=\bigcup_{i, j} I_{k+1, i, j}$, where intervals $I_{k+1, i, j}$ are closed and disjoint.

We may choose constants $a_{k, i, j}>0$ inductively so that every $g_{k}$ is continuous and $\sup \left\{x \in[0,1]:\left|g_{k}(x)-g_{k+1}(x)\right|\right\}<2^{-k}$ for every $k$. If $0 \leq x \leq 1$, we define $g(x)=\lim _{k \rightarrow \infty} g_{k}(x)$ and $f(x)=\int_{0}^{x} g$. If $x \in I_{l-1, i, j}^{\prime} \cap C_{l}$ and $y \in I_{l-1, i, j}^{\prime}$ with $y>x$, we have $g(z) \geq b_{l-1, i, j}$ for all $z \in(x, y)$ and $g>b_{l-1, i, j}$ in a set of positive 


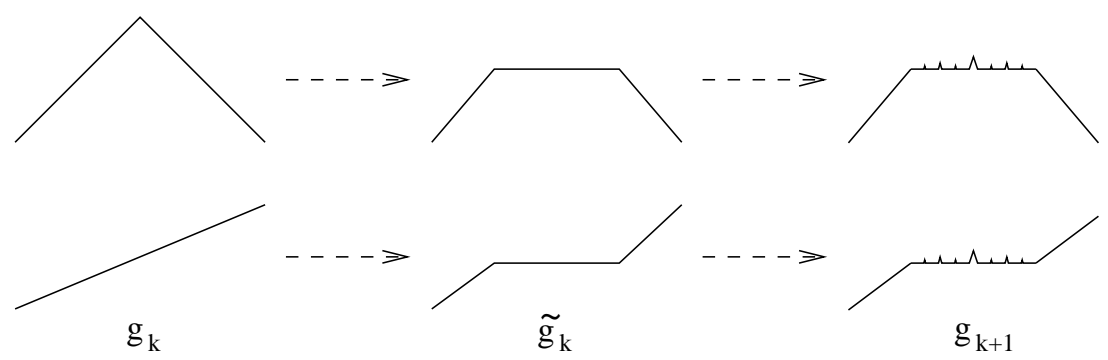

Figure 1. Construction of $g_{k+1}$ from $g_{k}$ on $I_{k, i, j}$.

measure on $(x, y)$. This implies

$$
f(y)=f(x)+\int_{x}^{y} g(t) d t>f(x)+b_{l-1, i, j}(y-x)=f(x)+f^{\prime}(x)(y-x) .
$$

If $y<x$, then the above inequality is switched. Thus, if $x \in C_{l}$, and $r=r(x)>0$ is such that $(x-r, x+r) \subset I_{l-1, i, j}^{\prime}$ for some $i$ and $j$, then properties (2.1) and (2.2) are valid. Since $\mathcal{L}\left((0,1) \backslash \bigcup_{l} C_{l}\right)=0$ and for any $x \in \bigcup_{l} C_{l}$, there is $I_{l-1, i, j}^{\prime}$ such that $x \in C_{l} \cap I_{l-1, i, j}^{\prime}$ (we put $I_{0,1,1}^{\prime}=(0,1)$ ), the construction is completed.

Problems. 1. In the above example, the set where conditions (2.1) and (2.2) hold seems to be a first category set. Is it possible for a $C^{1}$-function, for these properties to hold on a residual subset of $(0,1)$ ? If not, then what is the case if we require only that both of the sets $A^{+}(f, x)$ and $A^{-}(f, x)$ have positive lower density at $x$ in a residual set?

2. Are there functions $f \in C^{1}[0,1]$ so that both of the one-sided lower densities,

$$
\begin{aligned}
& \liminf _{r \downarrow 0} \mathcal{L}\left((x, x+r) \cap A^{+}(f, x)\right) / r, \\
& \liminf _{r \downarrow 0} \mathcal{L}\left((x, x+r) \cap A^{-}(f, x)\right) / r,
\end{aligned}
$$

are strictly positive in a set of positive (or even full) measure? Similar questions can be posed when intervals $(x, x+r)$ are replaced by some other sets.

2.2. Typical behavior. Given a property for functions, it is natural to ask if this property is typical on a function class in question. The theme of this section is to study the typicality of some properties related to the above example. For $k \in \mathbb{N} \cup\{0, \infty\}$, the space $C^{k}[0,1]$ is given the norm $\|f\|=\sup \left\{\left|f^{j}(x)\right|: x \in\right.$ $(0,1), j \in \mathbb{N}, 0 \leq j \leq k\}$ where $f^{0}=f$. When we say that some property holds for a typical $f \in C^{k}[0,1]$ we mean that this property is valid on a residual set of functions on $C^{k}[0,1]$. 
Some very basic facts are listed in the proposition below. To help discussion, we write for $f \in C^{1}[0,1]$,

$$
\begin{gathered}
A_{f}=\left\{x \in(0,1): x \in \overline{A^{+}(f, x)} \bigcap \overline{A^{-}(f, x)}\right\}, \\
B_{f}^{+}=\{x \in(0,1): \text { conditions }(2.1) \text { and }(2.2) \text { hold for some } r>0\}, \\
B_{f}^{-}=B_{-f}^{+} .
\end{gathered}
$$

Proposition 2.2. (1) For any $f \in C^{2}[0,1]$, the set $A_{f}$ is nowhere dense.

(2) If $2 \leq k \in \mathbb{N} \cup\{\infty\}$, then $\mathcal{L}\left(A_{f}\right)=0$ for a typical $f \in C^{k}[0,1]$.

(3) The set $(0,1) \backslash A_{f}$ is dense for any $f \in C^{1}[0,1]$.

(4) The sets $B_{f}^{+}$and $B_{f}^{-}$are dense for a typical $f \in C^{1}[0,1]$.

Proof. If $f \in C^{2}[0,1]$ and $x \in(0,1)$ is such that both of the sets $(x-r, x+$ $r) \cap A^{+}(f, x)$ and $(x-r, x+r) \cap A^{-}(f, x)$ are nonempty for any $r>0$, then $f^{\prime \prime}(x)=0$. This implies (1). Also (2) follows since $\mathcal{L}\left(\left\{x: f^{\prime \prime}(x)=0\right\}\right)=0$ for a typical $f \in C^{k}[0,1]$ when $k \geq 2$. Claim (3) follows from the mean value theorem, and (4) is true because the derivative of a typical $C^{1}$-function has a dense set of minima and maxima.

A natural question arising from our example is the following: Is it true that for a typical $f \in C^{1}[0,1]$, both of the sets $A^{+}(f, x)$ and $A^{-}(f, x)$ have strictly positive lower density at $x$ for almost every $x \in(0,1)$. This is, however, not the case; As we shall see, both of these sets have typically unit upper density and thus also zero lower density for almost every $x$. We shall prove this by using the following generalization of a well known theorem of Jarnik [2]. We say that $c \in \overline{\mathbb{R}}$ is a symmetrical essential derived number of $f:[0,1] \rightarrow \mathbb{R}$ at $x \in(0,1)$, denote $c \in \operatorname{SEDN}(f, x)$, if there is a set $E=E(x, c) \subset \mathbb{R}$ such that $E$ has unit upper density at $x$ and

$$
\lim _{\substack{y \in E \\ y \rightarrow x}} \frac{f(y)-f(x)}{y-x}=c .
$$

To avoid confusion, we note that in this context the term "symmetrical" does not refer to symmetric differentiation but to symmetrical upper density.

Theorem 2.3. For a typical $f \in C[0,1]$, we have $\operatorname{SEDN}(f, x)=\overline{\mathbb{R}}$ for almost every $x \in(0,1)$.

Remarks. A number $c \in \bar{R}$ is called a right essential derived number of $f$ at $x$ if there is $E \subset \mathbb{R}$ satisfying (2.5) with $\lim \sup _{r \downarrow 0} \mathcal{L}((x, x+r) \cap E) / r=1$. Left essential derived numbers are defined in an analogous way. A point $x \in(0,1)$ is an essential knot point of $f$ if every $c \in \bar{R}$ is simultaneously left and right essential derived number of $f$ at $x$. Jarnik [2] proved that almost all points are essential knot points for a typical function $f \in C[0,1]$. The above theorem is stronger than Jarnik's result since it allows us to choose $E$ such that it is 
simultaneously big at both sides of $x$ for some small scales, and not only big at left for some scales and big at right for some (possibly different) scales.

If $w \in \mathbb{R}^{2}$ and $c, \alpha \in \mathbb{R}$, we denote by $\ell_{w, c}$ the line through $w$ with slope $c$ and put $X(w, c, \alpha)=\left\{v \in \mathbb{R}^{2}: d\left(v-w, \ell_{w, c}\right) \leq \alpha|v-w|\right\}$. These cones are useful since $c \in \operatorname{SEDN}(f, x)$ if and only if

$$
\begin{aligned}
& x \in \bigcap_{\epsilon, \alpha, r_{0}}\left\{z \in(0,1): \exists 0<r<r_{0}\right. \text { such that } \\
& \quad \mathcal{L}(\{y \in(z-r, z+r):(y, f(y)) \in X((z, f(z)), c, \alpha)\})>(2-\epsilon) r\}
\end{aligned}
$$

where the intersection is taken over all positive rationals $\epsilon, \alpha$ and $r_{0}$, see also [9, Lemma 1].

Proof of Theorem 2.3. For $f \in C[0,1]$, let

$$
F=F(f)=\{x \in(0,1): \operatorname{SEDN}(f, x) \neq \overline{\mathbb{R}}\} .
$$

It follows from (2.6) that

$$
\begin{aligned}
F=\bigcup_{c, \epsilon, \alpha, r_{0}}\{x \in(0,1): \mathcal{L}(\{y \in(x-r, x+r): \\
\left.\quad(y, f(y)) \notin X((x, f(x)), c, \alpha)\})>\epsilon r \text { for all } 0<r<r_{0}\right\}
\end{aligned}
$$

where the union is taken over all $c \in \mathbb{Q}$, and $0<\epsilon, \alpha, r_{0} \in \mathbb{Q}$. Thus

$$
\{f \in C[0,1]: \mathcal{L}(F(f))>0\}=\bigcup_{\delta, c, \epsilon, \alpha, r_{0}} \mathcal{A}\left(\delta, c, \epsilon, \alpha, r_{0}\right)
$$

where $c \in \mathbb{Q}, 0<\delta, \epsilon, \alpha, r_{0} \in \mathbb{Q}$, and $\mathcal{A}\left(\delta, c, \epsilon, \alpha, r_{0}\right) \subset C[0,1]$ is given by

$$
\begin{aligned}
\mathcal{A}\left(\delta, c, \epsilon, \alpha, r_{0}\right)= & \{f \in C[0,1]: \mathcal{L}(\{x \in(0,1): \mathcal{L}(\{y \in(x-r, x+r): \\
& \left.\left.\left.(y, f(y)) \notin X((x, f(x)), c, \alpha)\})>\epsilon r \text { for all } 0<r<r_{0}\right\}\right)>\delta\right\} .
\end{aligned}
$$

Fix numbers $c \in \mathbb{R}$, and $\delta, \epsilon, \alpha, r_{0}>0$. It suffices to prove that the set $\mathcal{A}\left(\delta, c, \epsilon, \alpha, r_{0}\right)$ is nowhere dense on $C[0,1]$. Take $f \in C[0,1]$ and let $0<r<r_{0}$. Let $g \in C[0,1]$ with $\|f-g\|<r / 2$ so that it satisfies the following (see [8, Lemma 1]): There are disjoint intervals $I_{1}, \ldots, I_{k} \subset(0,1)$ with $\sum_{i=1}^{k} \ell\left(I_{i}\right)>1-\delta / 2$ such that $g$ is affine and has slope $c$ on each interval $I_{i}$. Let $0<\ell<\min _{i=1, \ldots, k} \ell\left(I_{i}\right)$, $0<t<\min \left\{\delta / 4, r_{0}\right\}$, and $0<s<\min \{\alpha \epsilon t / 4, r / 2\}$. Take $h \in B(g, s \ell)$. It is easy to see that if $x \in I_{i}$ with $d\left(x, \partial I_{i}\right)>t \ell$, then $(y, h(y)) \in X((x, h(x)), c, \alpha)$ for all $y \in(x-t \ell, x+t \ell) \backslash(x-2 s \ell / \alpha, x+2 s \ell / \alpha)$. It follows that for such $x$, we have

$$
\mathcal{L}(\{y \in(x-t \ell, x+t \ell):(y, h(y)) \notin X((x, h(x)), c, \alpha)\})<4 s \ell / \alpha<t \ell \epsilon .
$$

Since (2.7) holds in a measurable set whose measure is greater than $1-\delta / 2-$ $k 2 t \ell>1-\delta$, we conclude that $h \notin \mathcal{A}\left(\delta, c, \epsilon, \alpha, r_{0}\right)$. Thus $B(g, s \ell) \subset B(f, r) \backslash$ $\mathcal{A}\left(\delta, c, \epsilon, \alpha, r_{0}\right)$ and the claim follows. 
Zajíček [8] strengthened Jarnik's result using porosity notations. His result is also in a sense one-sided and does not seem to imply Theorem 2.3. However, only a minor change in his method gives even stronger theorem. The following notation is from [8]. If $A \subset \mathbb{R}$ and $I \subset \mathbb{R}$ is an interval, the number $p(A, I)$ denotes the length of the largest subinterval $I^{\prime} \subset I \backslash A$. We denote by $\mathcal{G}$ the collection of all increasing functions $g$ on $(0, \infty)$ for which $g(x)>x$ for all $0<x<$ $\infty$. If $g \in \mathcal{G}$, we say that $E \subset \mathbb{R}$ is $[g]$-porous from the right (left) at $x \in \mathbb{R}$ if there is a sequence $r_{i} \downarrow 0$ such that $g\left(p\left(E,\left(x, x+r_{i}\right)\right)\right)>r_{i}\left(g\left(p\left(E,\left(x-r_{i}, x\right)\right)\right)>r_{i}\right)$ for all $i \in \mathbb{N}$. A number $c \in \overline{\mathbb{R}}$ is a right (left) $[g]$-derived number of $f$ at $x$ if there is a set $E \subset \mathbb{R}$ for which $\mathbb{R} \backslash E$ is $[g]$-porous from the right (left) at $x$ such that (2.5) holds for $E$. A point $x$ is a $[g]$-knot point of $f$ if every $c \in \overline{\mathbb{R}}$ is both left and right $[g]$-derived number of $f$ at $x$. Zajíček [8, Theorem 2] showed that for a typical $f \in C[0,1]$, the set of points from $(0,1)$ which are not $[g]$-knot points of $f$ is $\sigma$ - $[g]$-totally porous. See [8, Definition 2] or [7, Definition 2.49] for the definition of $[g]$-totally porous sets.

Modifying the above notation, we say that $A \subset \mathbb{R}^{n}$ is symmetrically $[g]$-porous at $x$ if there is a sequence $r_{i} \downarrow 0$ so that $\min \left\{g\left(p\left(A,\left(x-r_{i}, x\right)\right)\right), g(p(A,(x, x+\right.$ $\left.\left.\left.r_{i}\right)\right)\right)>r_{i}$ for each $i \in \mathbb{N}$. A number $c \in \overline{\mathbb{R}}$ is a symmetrical $[g]$-derived number of $f$ at $x$ if there is a set $E \subset \mathbb{R}$ such that $\mathbb{R} \backslash E$ is symmetrically $[g]$-porous at $x$ and (2.5) holds for $E$. A point $x$ is a symmetrical $[g]$-knot point of $f$ if each $c \in \overline{\mathbb{R}}$ is a symmetrical $[g]$-derived number of $f$ at $x$.

Theorem 2.4. Let $g \in \mathcal{G}$. Then for a typical $f \in C[0,1]$, the set of points $x \in(0,1)$ which are not symmetrical $[g]$-knot points of $f$ is $\sigma$ - $[g]$-totally porous.

This theorem can be proved by modifying Zajíček's method only slightly and thus we shall not repeat the argument. For the convenience of an interested reader we comment that the main point is that in $[8$, Lemma 2(b)] one can assert: For any $h \in U(a, b, s, \delta)$ and $x \in \cup_{k=0}^{n-1}[k / n+2 v,(k+1) / n-2 v]$ the inequalities $g(p(\{y:(h(y)-h(x)) /(y-x) \notin[a, b]\},[x, x+v]))>v$ and $g(p(\{y$ : $(h(y)-h(x)) /(y-x) \notin[a, b]\},[x-v, x]))>v$ hold.

We now turn our attention back to our original question related to the distribution of the sets $A^{+}(f, x)$ and $A^{-}(f, x)$ for typical functions $f \in C^{1}[0,1]$.

Theorem 2.5. For a typical $f \in C^{1}[0,1]$, both of the sets $A^{+}(f, x)$ and $A^{-}(f, x)$ have unit upper density and zero lower density at $x$ for almost every $x \in(0,1)$.

Proof. By symmetry, it suffices to show that

$$
\limsup _{r \downarrow 0} \mathcal{L}\left((x-r, x+r) \cap A^{+}(f, x)\right) /(2 r)=1
$$

almost everywhere for a typical $f \in C^{1}[0,1]$. In fact, we shall verify a stronger statement according to which for typical $f \in C^{1}[0,1]$ the set $(0,1) \backslash A^{+}(f, x)$ is strongly symmetrically porous (see $[7$, p. 320]) at $x$ for almost every $x \in(0,1)$. Using Theorem 2.3, we see that for typical $f \in C^{1}[0,1]$, we have $\operatorname{SEDN}\left(f^{\prime}, x\right)=\overline{\mathbb{R}}$ 
almost everywhere. Fix such a function $f$ and $x \in(0,1)$ for which $\operatorname{SEDN}\left(f^{\prime}, x\right)=$ $\overline{\mathbb{R}}$. Now there is $E \subset(0,1)$ with

$$
\limsup _{r \downarrow 0} \mathcal{L}((x-r, x+r) \cap E) /(2 r)=1
$$

and

$$
\lim _{\substack{t \in E \\ t \rightarrow x}} \frac{f^{\prime}(t)-f^{\prime}(x)}{t-x}=2 .
$$

Thus we may choose $0<r_{0}<1$ so that

$$
\left(f^{\prime}(t)-f^{\prime}(x)\right) /(t-x)>1 \text { for all } t \in(x-r, x+r) \cap E \backslash\{x\} .
$$

Replacing $E$ by it's closure if necessary, we may assume that it is measurable.

Choose $0<M<\infty$ so that $\left|f^{\prime}(x)\right|<M$ for all $x \in[0,1]$ and let $\varepsilon>0$ be such that $1-(4 M+2) \varepsilon>0$. Using (2.8), we may find arbitrary small radii $0<r<r_{0}$ such that $\mathcal{L}((x-r, x+r) \cap E)>\left(2-\varepsilon^{2}\right) r$. If $y \in(x+\varepsilon r, x+r)$ for such a radius $r$, then the above facts and (2.9) imply

$$
\begin{aligned}
\int_{x}^{y} f^{\prime} d \mathcal{L} & =\int_{(x, y) \cap E} f^{\prime} d \mathcal{L}+\int_{(x, y) \backslash E} f^{\prime} d \mathcal{L} \\
& >\int_{(x, y) \cap E} f^{\prime}(x) d t+\int_{(x, y) \cap E}(t-x) d t-\varepsilon^{2} M r \\
& >f^{\prime}(x)(y-x)-\varepsilon^{2} r M+\varepsilon r / 2-\varepsilon^{2} r-\varepsilon^{2} M r \\
& =f^{\prime}(x)(y-x)+(1-(4 M+2) \varepsilon) \varepsilon r / 2 \\
& >f^{\prime}(x)(y-x) .
\end{aligned}
$$

Thus $f(y)=f(x)+\int_{x}^{y} f^{\prime} d \mathcal{L}>f(x)+f^{\prime}(x)(y-x)$ giving $y \in A^{+}(f, x)$. In a similar manner, we see that $y \in A^{+}(f, x)$ also if $y \in(x-r, x-\varepsilon r)$. Letting $\varepsilon \downarrow 0$ gives the claim.

Remark. Using Theorem 2.4 in place of Theorem 2.3 in the above proof leads to stronger conclusions. For example, if $g \in \mathcal{G}$, then we see that for a typical $f \in C^{1}[0,1]$, both of the sets $(0,1) \backslash A^{+}(f, x)$ and $(0,1) \backslash A^{-}(f, x)$ are strongly symmetrically porous at $x$ for a set of points $x$ whose complement is $\sigma$ - $[g]$-totally porous.

\section{Conical Densities}

Let $f$ and $C_{l}, l \in \mathbb{N}$, be defined as in the proof of Theorem 2.1. Define $\mathcal{G}=\{(t, f(t)): t \in(0,1)\}$ and $\mathcal{G}_{C}=\left\{(t, f(t)): t \in \bigcup_{l} C_{l}\right\}$.

Fix $x=(t, f(t)) \in \mathcal{G}_{C}$. It is clear that

$$
\liminf _{r \downarrow 0} \mathcal{H}^{1}(B(x, r) \cap \mathcal{G} \cap H(x, \theta)) / r>0
$$


whenever $\theta \in S^{1}$ is not perpendicular with the tangent of $\mathcal{G}$ at $x$. Therefore we consider only directions of the form $\theta= \pm\left(-f^{\prime}(t), 1\right) /\left(1+f^{\prime}(t)^{2}\right)^{1 / 2}$. If $r>0$ is small, then $(s, f(s)) \in B(x, r)$ for $s \in(t-c r, t+c r)$ and $c=\left(1+2 f^{\prime}(t)^{2}\right)^{-1 / 2}$. It follows from $(2.1)-(2.2)$ that $\mathcal{H}^{1}(B(x, r) \cap \mathcal{G} \cap H(x, \theta)) \geq c r$. We conclude that (3.1) holds for all $x \in \mathcal{G}_{C}$ and $\theta \in S^{1}$. This implies that claim (2) of Theorem 1.1 is not always valid for rectifiable curves.

By modifying the above example, one can easily construct $m$-rectifiable surfaces $S$ on $\mathbb{R}^{n}$, for any integer $1<m<n$, so that

$$
\liminf _{r \downarrow 0} r^{-m} \mathcal{H}^{m}(B(x, r) \cap H(x, \theta) \cap S)>0
$$

for any $\theta \in S^{n-1}$ and for $\mathcal{H}^{m}$-almost all $x \in S$. One can take, for example, $S$ to be the graph of the function

$$
g:(0,1) \times \mathbb{R}^{m-1} \rightarrow \mathbb{R}^{n-m}:\left(x_{1}, \ldots, x_{m}\right) \mapsto\left(f\left(x_{1}\right), \ldots, f\left(x_{1}\right)\right),
$$

where $f$ is as above.

Marstrand's argument from [4, pp. 293-297] can be generalized to prove that in $\mathbb{R}^{n}$ claim (1) of Theorem 1.1 holds for $0<s<1$, and claim (2) for $n-1<s<n$, see also [5, Theorem 11.11]. For general $0<s<n$, the following is known, see Lorent [3] and Suomala [6]. If $m \in \mathbb{N}$, then a set $A \subset \mathbb{R}^{n}$ is called $m$-rectifiable if $\mathcal{H}^{m}$-almost all of it can be covered with a countable union of $C^{1}$-images of $\mathbb{R}^{m}$. A set $A$ is called purely $m$-unrectifiable, if it intersects every $C^{1}$-image of $\mathbb{R}^{m}$ only in a set of $\mathcal{H}^{m}$ measure zero.

Theorem 3.1. Let $A \subset \mathbb{R}^{n}$ with $\mathcal{H}^{s}(A)<\infty$ and let $V$ be an m-dimensional linear subspace of $\mathbb{R}^{n}$. If either $0<s<m$ or if $s=m$ and $A$ is purely $m$ unrectifiable, then for $\mathcal{H}^{s}$ almost every $x \in A$, there is $\theta \in V \cap S^{n-1}$ such that

$$
\liminf _{r \downarrow 0} r^{-s} \mathcal{H}^{s}(B(x, r) \cap H(x, \theta, \eta) \cap A)=0
$$

for any $\eta>0$.

The examples discussed above show that one cannot always take $\eta=0$ in (3.3) when $s \in[1, m) \cap \mathbb{N}$ and $A$ is $s$-rectifiable. On the other hand, Besicovitch's argument from [1, pp. 317-320] can be modified to prove that even claim (1) of Theorem 1.1 holds for any purely 1-unrectifiable set $A \subset \mathbb{R}^{n}$ with $\mathcal{H}^{1}(A)<\infty$.

Problem. Does Theorem 3.1 hold with $\eta=0$ when either $s \in(1, n-1)$ is nonintegral, or $s \in[2, n-1] \cap \mathbb{N}$ and $A$ is purely $s$-unrectifiable?

\section{REFERENCES}

[1] A.S. Besicovitch. On the fundamental geometrical properties of linearly measurable plane sets of points II. Math. Ann., 115:296-329, 1938.

[2] V. Jarník. Sur les nombres dérivés approximatifs. Fund. Math., 22:4-16, 1934.

[3] A. Lorent. A generalised conical density theorem for unrectifiable sets. Ann. Acad. Sci. Fenn. Math., 28(2):415-431, 2003. 
[4] J.M. Marstrand. Some fundamental geometrical properties of plane sets of fractional dimensions. Proc. London Math. Soc. (3), 4:257-301, 1954.

[5] P. Mattila. Geometry of Sets and Measures in Euclidean Spaces: Fractals and rectifiability. Cambridge University Press, Cambridge, 1995.

[6] V. Suomala. On the conical density properties of measures on $\mathbb{R}^{n}$. Math. Proc. Cambridge Philos. Soc. to appear.

[7] L. Zajíček. Porosity and $\sigma$-porosity. Real Anal. Exchange, 13(2):314-350, 1987/88.

[8] L. Zajíček. Porosity, derived numbers and knot points of typical continuous functions. Czechoslovak Math. J., 39(114)(1):45-52, 1989.

[9] L. Zajíček. On essential derived numbers of typical continuous functions. Tatra Mt. Math. Publ., 2:123-125, 1993.

Department of Mathematics and Statistics P.O. Box 35 (MaD), FiN-40014 UniVERSITY OF JYVÄSKYLÄ, FINLAND,

E-mail address: visuomal@maths.jyu.fi 